\title{
Double intrahepatic bile duct puncture: a pitfall during endoscopic ultrasound- guided hepaticogastrostomy
}

Endoscopic ultrasound-guided hepaticogastrostomy (EUS-HGS) has emerged as an alternative technique to endoscopic retrograde cholangiopancreatography (ERCP) $[1,2]$. During EUS-HGS, the process of fistula dilation is technically challenging. To date, various dilation devices

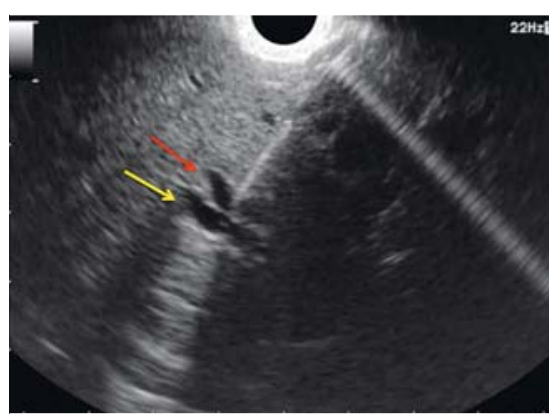

- Fig. 1 Endoscopic ultrasound image showing a double intrahepatic bile duct (red arrow, proximal bile duct; yellow arrow, distal bile duct). and techniques have been reported [35]. However, despite improvements in dilation devices, challenging cases are still sometimes encountered. We herein describe a pitfall of EUS-HGS, which may be one of the reasons for the challenges encountered in some cases.

The presented case was a 78-year-old woman with advanced cholangiocarcinoma causing duodenal and biliary obstruction. Duodenal metal stent placement was first performed as an endoscopic retrograde cholangiopancreatography (ERCP) failed because of duodenal obstruction. Next, an echoendoscope was inserted into the stomach and the intrahepatic bile duct was punctured using a 19G fine needle aspiration (FNA) needle. However, because the axis between the bile duct and FNA needle was slightly misaligned, double intrahepatic bile duct occurred ( $\triangleright$ Fig. 1 ), a phenomenon that was confirmed on cholangiog- raphy after the injection of contrast medium ( $\triangleright$ Fig. 2 a).

A 0.025-inch guidewire was inserted into the biliary tract and a fine gauge balloon catheter (REN; Kaneka, Osaka, Japan) was inserted to dilate the fistula, as previously described [5]. However, as the balloon catheter could not be inserted into the biliary tract, a diathermic dilator was used ( Fig.2 b). Although this device could be passed through the proximal intrahepatic bile duct, we could not insert it into the common bile duct. Therefore, balloon catheter insertion was again attempted, following which it was then possible to insert the device through the double intrahepatic bile duct ( $\triangleright$ Fig. $\mathbf{2 c}$ ) and fistula dilation was successfully performed ( $\triangleright$ Fig.2d). Finally, stent placement using a covered metal stent $(10 \mathrm{~mm} \times 12 \mathrm{~cm}$, Niti-S Biliary Covered Stent; Taewoong Medical, Seoul, South Korea) was successfully
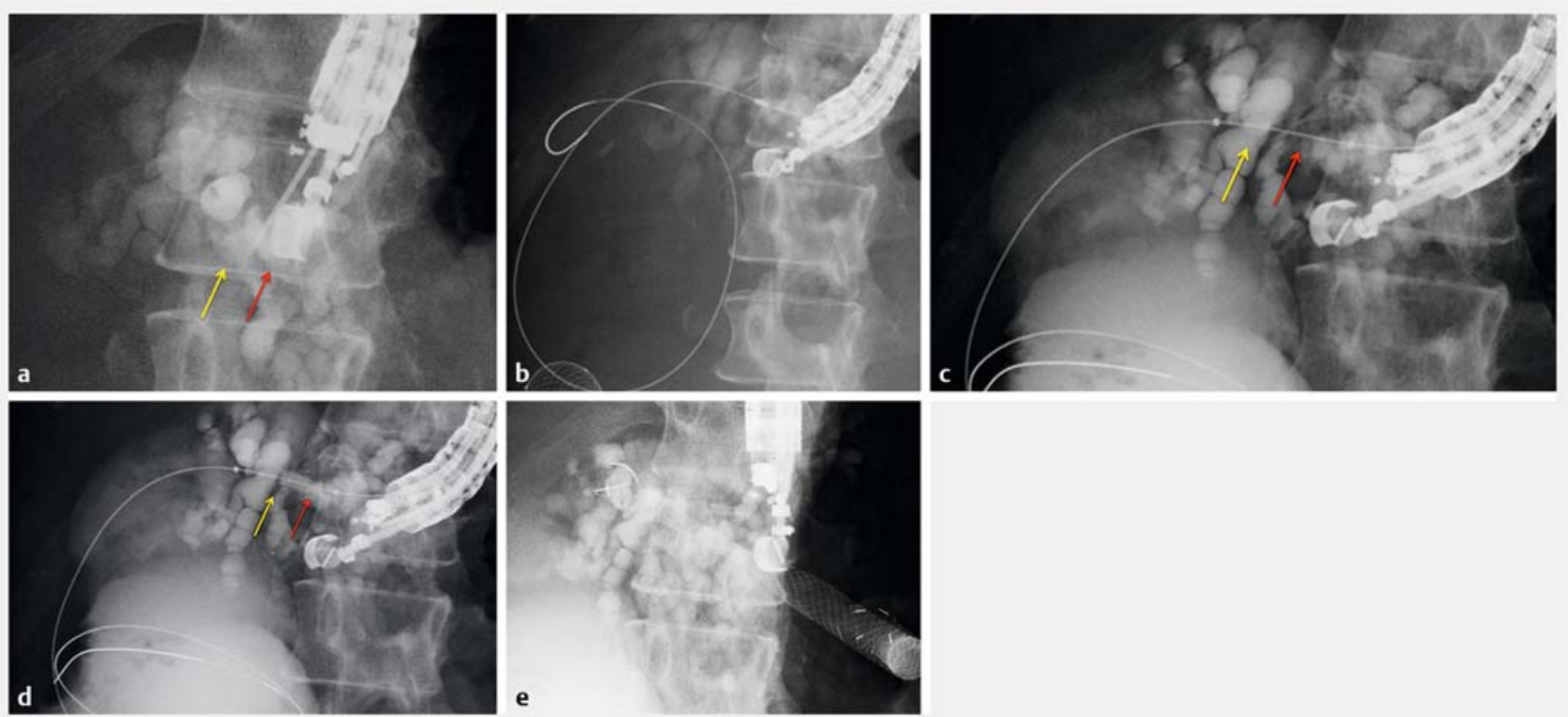

Fig. 2 Cholangiographic images (red arrow, proximal bile duct; yellow arrow, distal bile duct) showing: a the double intrahepatic bile duct following contrast injection; $\mathbf{b}$ inability to insert the balloon catheter into the biliary tract, despite the use of a diathermic dilator, meaning fistula dilation was challenging; $\mathbf{c}$ the intrahepatic bile duct being dilated again using a balloon catheter; $\mathbf{d}$ the notch caused by the double intrahepatic bile duct; e the covered metal stent that was placed to successfully perform endoscopic ultrasound-guided hepaticogastrostomy. 


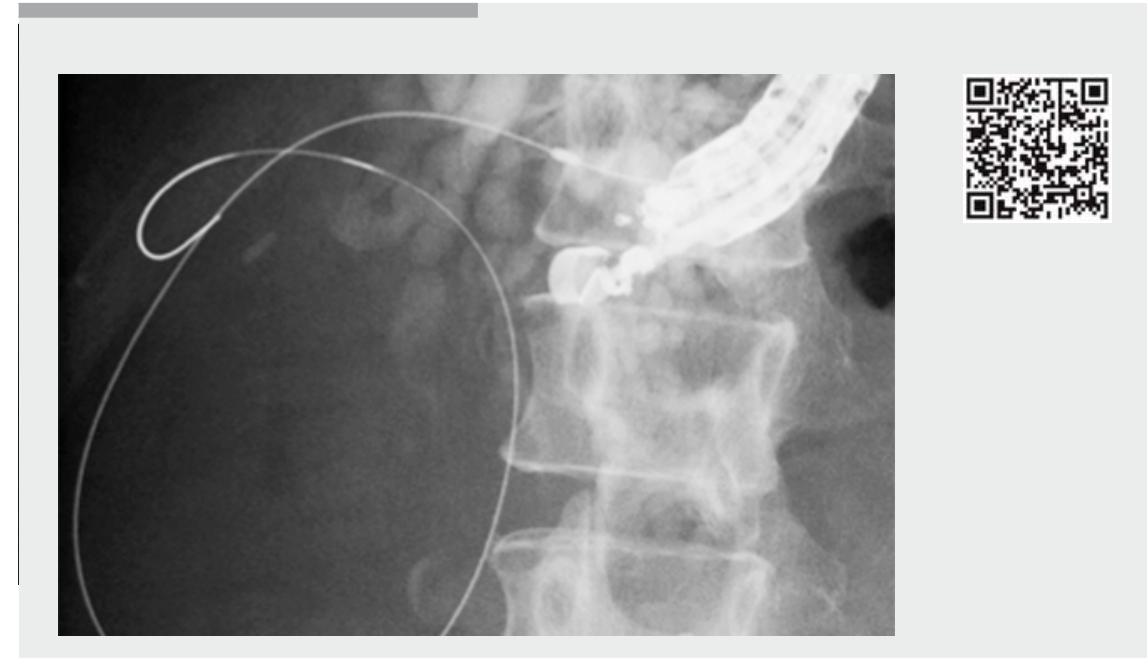

Video 1 The intrahepatic bile duct was punctured using a 19G fine needle aspiration (FNA) needle; however, because the axis between the bile duct and the FNA needle was slightly misaligned, double intrahepatic bile duct occurred. After contrast medium had been injected, the guidewire was inserted into the biliary tract. Although a fine gauge balloon catheter was used, it was not possible to insert the device into the biliary tract. Fistula dilation using a diathermic dilator was therefore attempted; however, although this device could be passed through the proximal intrahepatic bile duct, it could not be inserted into the common bile duct. Balloon catheter insertion was then attempted again and this time it was possible to insert the device through the double intrahepatic bile duct, allowing EUS-HGS finally to be successfully performed.

performed ( $>$ Fig. 2 e; $>$ Video 1 ). Unfortunately, because of the prolonged procedure time (58 minutes), biliary peritonitis occurred, which subsequently required 7 days of conservative treatment.

Our experience suggests that if a double intrahepatic bile duct is encountered during EUS-HGS, re-puncture for the second bile duct may be considered.

Endoscopy_UCTN_Code_CPL_1AL_2AD

\section{Competing interests}

None

\section{References}

[1] Rimbas M, Larghi A. Endoscopic ultrasonography-guided techniques for accessing and draining the biliary system and the pancreatic duct. Gastrointest Endosc Clin N Am 2017; 27: $681-705$

[2] Ogura T, Higuchi K. Technical tips for endoscopic ultrasound-guided hepaticogastrostomy. World J Gastroenterol 2016; 22: $3945-3951$

[3] Dhir V, Isayama H, Itoi T et al. Endoscopic ultrasonography-guided biliary and pancreatic duct interventions. Dig Endosc 2017; 29: $472-485$

[4] Law R, Baron TH. Endoscopic ultrasoundguided biliary interventions: an update on recent developments. Curr Opin Gastroenterol 2016; 32: 232-237

[5] Amano M, Ogura T, Onda S et al. Prospective clinical study of endoscopic ultrasoundguided biliary drainage using novel balloon catheter (with video). J Gastroenterol Hepatol 2017; 32: $716-720$

\section{Bibliography}

DOI https://doi.org/10.1055/a-0605-2710

Published online: 7.6.2018

Endoscopy 2018; 50: E195-E196

(c) Georg Thieme Verlag KG

Stuttgart $\cdot$ New York

ISSN 0013-726X

The authors

Takeshi Ogura, Atsushi Okuda, Akira Miyano, Rieko Kamiyama, Nobu Nishioka, Kazuhide Higuchi

2nd Department of Internal Medicine, Osaka

Medical College, Osaka, Japan

\section{Corresponding author}

\section{Takeshi Ogura, MD}

2nd Department of Internal Medicine, Osaka Medical College, 2-7 Daigakuchou,

Takatsukishi, Osaka 569-8686, Japan Fax: $+81-726846532$

oguratakeshi0411@yahoo.co.jp

\section{ENDOSCOPY E-VIDEOS \\ https://eref.thieme.de/e-videos}

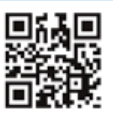

Endoscopy E-Videos is a free access online section, reporting on interesting cases and new

techniques in gastroenterological endoscopy. All papers include a high quality video and all contributions are freely accessible online.

This section has its own submission website at https://mc.manuscriptcentral.com/e-videos 